\title{
A survey of prescription pattern of anti-diabetic drugs on diabetic patients with cardiovascular complications within Dhaka metropolis
}

\author{
Rubaba Karim ${ }^{1}$, Rita Saha ${ }^{1}$, M. Sohanur Rahman ${ }^{2}$, Akhlatun Nure ${ }^{3}$, Khadija Akter Etu ${ }^{1}$, \\ Umme Jamila ${ }^{1}$, Taslima Begum ${ }^{1}$, Ajharul Islam ${ }^{4}$, Bikash Chandra Adhikary ${ }^{1}$, \\ Mst. Marium Begum ${ }^{*}$
}

\begin{abstract}
${ }^{1}$ Department of Pharmacy, Primeasia University, Dhaka1213, Bangladesh

${ }^{2}$ Department of Biochemistry and Molecular Biology, University of Rajshahi, Rajshahi-6205, Bangladesh ${ }^{3}$ Department of Zoology, University of Rajshahi, Rajshahi-6205, Bangladesh ${ }^{4}$ Department of Pharmacy, Dhaka International University, Banani-1213, Dhaka, Bangladesh

${ }^{5}$ Department of Pharmaceutical Technology, Faculty of Pharmacy, University of Dhaka1000, Bangladesh
\end{abstract}

Received: 07 September 2016 Revised: 11 September 2016 Accepted: 12 October 2016

*Correspondence to: Mst. Marium Begum, Email: mariumpharm23@ gmail.com

Copyright: (C) the author(s), publisher and licensee Medip Academy. This is an openaccess article distributed under the terms of the Creative Commons Attribution NonCommercial License, which permits unrestricted noncommercial use, distribution, and reproduction in any medium, provided the original work is properly cited.

\section{INTRODUCTION}

Diabetes is a chronic disease which is globally increasing and it also considered as an epidemic. Millions of people are affected with a large negative impression by Diabetes mellitus that is considered as one of the common chronic diseases. ${ }^{1}$ At present, approximately $4 \%$ of the global

\begin{abstract}
Background: This survey study was designed to analyze the current prescription pattern of anti-diabetic drugs that used in diabetic patients with cardiovascular complications within Dhaka metropolis.

Methods: The present study has been conducted by out-patient department in a number of well-known general and specialized government and private hospitals in Dhaka, Bangladesh for 5 months. 1200 prescriptions and questionnaire were randomly evaluated for this present survey. A standard questionnaire was prepared, containing 17 different questions, to conduct the survey based on patient's demographic data such as which type of diabetes they have contained, age, gender, education and their self-assessment of health, disease history and medication.

Results: Among the patients involved in this study, there were 46\% (550) male and $54 \%$ (650) female between the ages of 30 and 75 years. Between 46 and 70 years, $19 \%$ male and $31 \%$ female were found to be diabetic indicating that in this age group female are mostly affected by diabetes. About 2020 antidiabetic drugs were prescribed by the physicians in which $30.43 \%$ contained single, $54.16 \%$ contained two and $15 \%$ contained more than two anti-diabetic drugs. Amongst antidiabetic medications, metformin was the most commonly prescribed drug which was given in $30.69 \%$ patients followed by glimepride $10.9 \%, 5.45 \%$ had glipizide, $1 \%$ pioglitazone and other drugs. A total of $7.43 \%$ drug was prescribed by fixed dose combinations. Highest percentage of male diabetic patients with hypertension (86\%), dyslipidemia (100\%) and other complications $(57 \%)$ was found at 75 years of age while no female patients were found under the same condition at the same age.

Conclusions: The findings can serve as a guide to choose the formulation and combination of anti-diabetic drugs in this part of the world before developing \& marketing any new drug. Therefore it is necessary to create better awareness among people, focus on rational use of anti-diabetic drugs and also motivate our physicians to prescribe the generic drugs.
\end{abstract}

Keywords: CVD, Dhaka, Diabetes, Hypoglycemic agents, Prescription population is suffering from Diabetes mellitus which is expected to reach $5.4 \%$ in $2025 .^{2}$ In 1985,30 million people had diabetes in worldwide which was estimated by the World Health Organization (WHO, 2006) and this number increased to 135 million by 1995 and reached 217 million in 2005 .WHO has predicted that by the year 2030 this number will increase to at least 366 million. ${ }^{3}$ 
Diabetes is a chronic and complex disease caused by the body's inability to produce or respond to the pancreatic hormone insulin that controls blood glucose levels. Diabetes happens when there is low or no insulin production or improper use of insulin. The prevalence of type 2 diabetes mellitus is increasing in both developing and developed country and the incidence of type 1 diabetes (T1D) is also increasing parallely in worldwide.

People with type 2 diabetes have a greater incidence of cardiovascular disease, cerebrovascular disease, and renal disease than the general population. The clinical diagnosis of diabetes is often indicated by the presence of symptoms such as polyuria, polydipsia, and unexplained weight loss, and is confirmed by measurement of abnormal hyperglycemia. Hypertension is a highly prevalent disease worldwide and very common among patients with diabetes. Approximately from 10 to $30 \%$ of T1D and $60 \%$ of T2D patients have hypertension. ${ }^{4}$ Approximately 74.5 million and 23.6 million adults respectively in the United States are affected by hypertension and diabetes, and approximately $75 \%$ of patients with diabetes have concomitant hypertension. ${ }^{5}$ On the other hand, in diabetes mellitus dyslipidemia is one of the major risk factors for cardiovascular disease. In diabetic patients dyslipidemia are a high plasma triglyceride concentration, low HDL cholesterol concentration and increased concentration of small dense LDL-cholesterol particles. The lipid changes associated with diabetes mellitus are attributed to increased free fatty acid flux secondary to insulin resistance. ${ }^{6}$

The stimulation of endogenous insulin secretion, enhancement of insulin action at the target tissues, inhibition of dietary starch and lipid degradation, and treatment with oral hypoglycemic agents are currently available therapeutic strategies for the treatment of this chronic metabolic disorder. ${ }^{7}$ Some anti-diabetic drugs that are used to treat diabetes mellitus with the exceptions of insulin, exenatide, and liraglutide, pramlintide, all are administered orally and are thus also called oral hypoglycemic agents or oral antihyperglycemic agents. ${ }^{8}$ These anti-diabetic drugs are the drugs that work to lower abnormally high glucose (sugar) levels in the blood, which are characteristic of the endocrine system disorder (diabetes mellitus).

\section{METHODS}

This study was performed using prescriptions and questionnaire of around 1200 patients suffering from diabetes and some of them associated with hypertension and dyslipidemia. Various age group patients and patients with different types of diabetes have been selected for the study. This study conducted for a period of 5 months from August 2015 to January 2016.

\section{Selection area}

Selecting proper area for survey is a crucial part for getting perfect data, which represent the actual condition. We emphasized on some general and specialized governmental hospitals. Our main focus was actually out patients of endocrinology department. Prescriptions of newly registered patients were studied.

\section{Sampling design}

A sampling design is a definite plan for obtaining a sample from a given area. It refers to the technique or the procedure, the researchers would adopt in selecting items for the sample. Patient's data such as the age, name, gender and data on prescribed drugs that include name of drug, dosage form, route of administration, most prescribed drug and so on were recorded on a customized data collection sheet in an approved manner. Each drug was counted only once without considering any change in the regimen.

\section{Questionnaire}

A standard questionnaire was prepared, containing 17 different questions, to conduct the survey based on patient's age, disease history and medication. Our total patients were 1200 from those hospitals. The questionnaire has been used to collect proper information about Diabetes Mellitus and its association with cardiovascular complications in Bangladeshi population. The information that were collected from this survey by using questionnaire was related to the patient demographic data such as which type of diabetes they have contained, age, gender, education and their self assessment of health. A number of criteria were considered for analyzing questionnaire that includes, duration since they were diagnosed with diabetes, time interval to check their glucose level in a year. The most significant part of the questionnaire involved whether the patient has been diagnosed with any cardiovascular complications along with diabetes and for how long. A part of the questionnaire also helped us to determine the concurrent administration of antidiabetic drugs and other drugs for cardiovascular complications by the patients specifying the name of drugs, dose and dosage forms. Moreover, this also involved question mentioning use of insulin injection by patients in the past weeks or months.

The questionnaire has been filled up by the individuals at different hospitals who have participated to share their health issues.

\section{Data processing and graphical representation}

Finally all the collected raw data were processed and represented to various aspects through MS-Excel. The results were expressed as proportions or as percentages. 


\section{RESULTS}

A total of 1200 prescriptions were assessed. The patients involved $46 \%$ (550) male and 54\% (650) female between the ages of 30 and 75 years. Between 46 and 70 years, $19 \%$ male and $31 \%$ female were found to be diabetic indicating that in this age group female are mostly affected by diabetes (Figure 1).

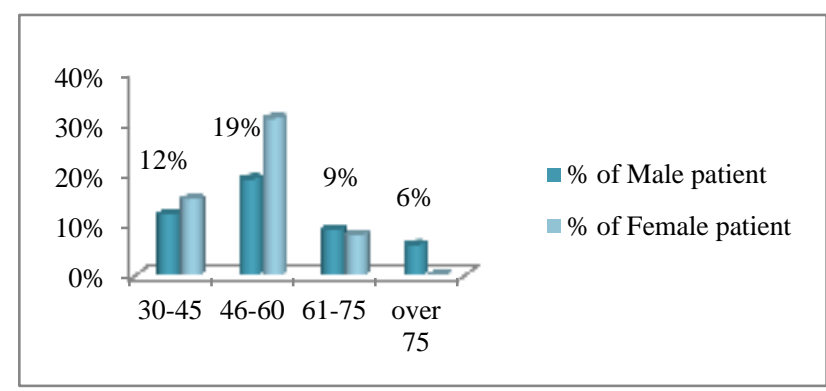

Figure 1: Percentage $(\%)$ of male and female patients affected by diabetes according their age group.

In the patient information section about 2020 drugs were prescribed. In the present case study, $30.83 \%$ prescriptions contained single anti-diabetic drug, $54.16 \%$ contained two anti-diabetic drugs and only $15 \%$ contained more than two anti-diabetic drugs (Figure 2).

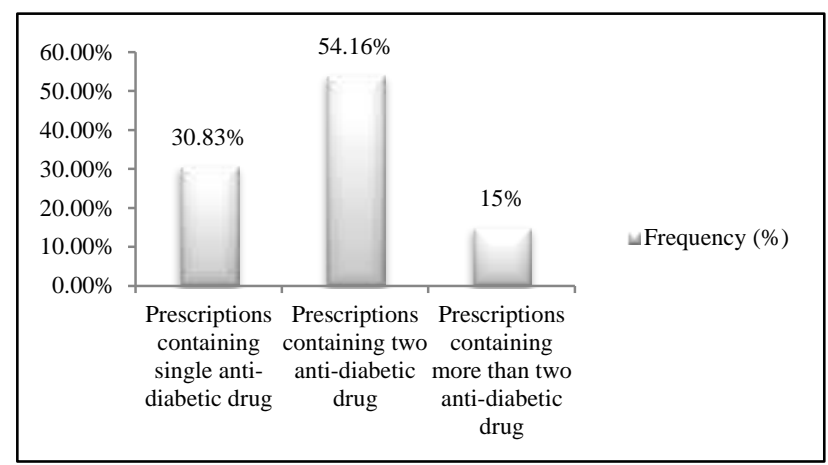

Figure 2: Proportion of prescriptions containing antidiabetic drug.

In Figure 3, it is shown that the percentage of the prescription written with either the generic or brand name. It was found that only $8.33 \%$ anti-diabetic drugs were prescribed by their generic name while the rest $(91.66 \%)$ were prescribed by brand names. Among these prescribed drugs, about $69.80 \%$ anti-diabetic drugs were solid dosage forms while $30.20 \%$ anti-diabetic drugs were injectables (Figure 4).

In these prescriptions, about $92.57 \%$ drugs were prescribed as single dose while $7.43 \%$ drugs were prescribed as fixed dose combinations where $30.3 \%$ Insulin, 30.69\% Metformin, 2.48\% Sitagliptin, 5\% Vildagliptin, 5\% Linagliptin, 2\% Glipizide, $5.45 \%$ Glimepiride, $10.9 \%$ Gliclazide and $1 \%$ Pioglitazone were found to be prescribed as single dose. However, it was observed that most commonly prescribed fixed dose combinations were $5.45 \%$ Vildagliptin + Metformin, $1.49 \%$ Sitagliptin + Metformin and $0.5 \%$ Pioglitazone + Metformin (Table 1).

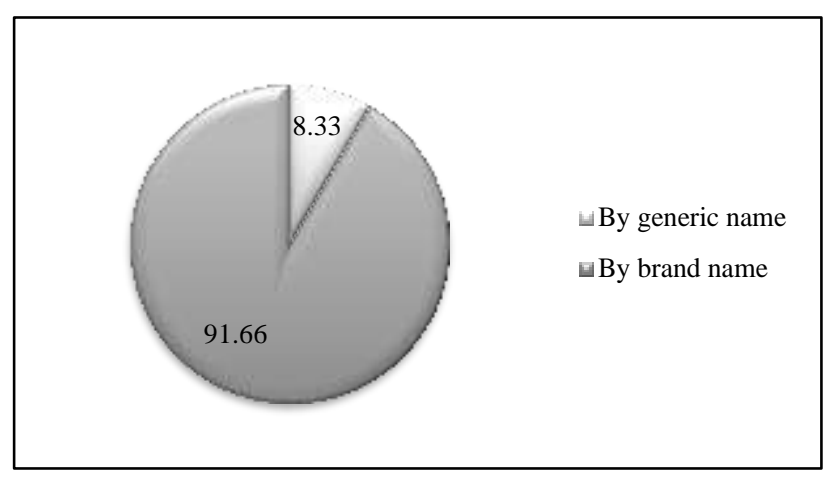

Figure 3: Percentage (\%) of anti-diabetic drugs prescribed by their generic or brand name.

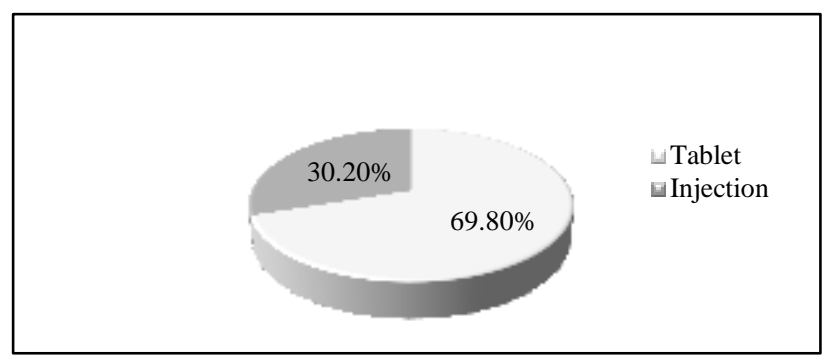

Figure 4: Percentage (\%) of anti-diabetic drugs prescribed by orally or parenterally.

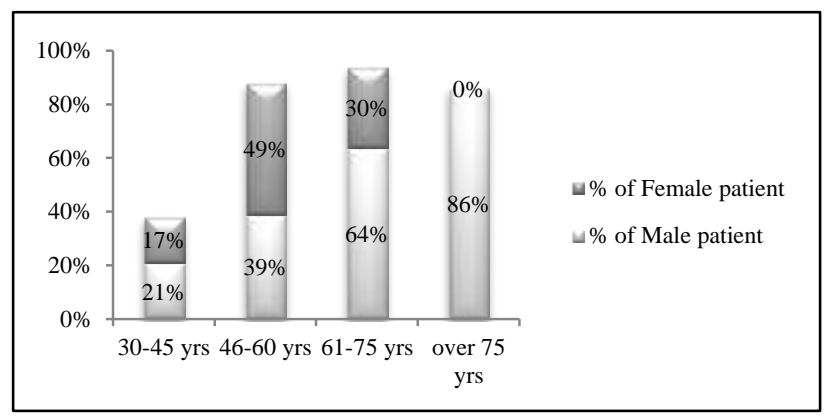

Figure 5: Percentage (\%) of patient of Diabetes associated with Hypertension.

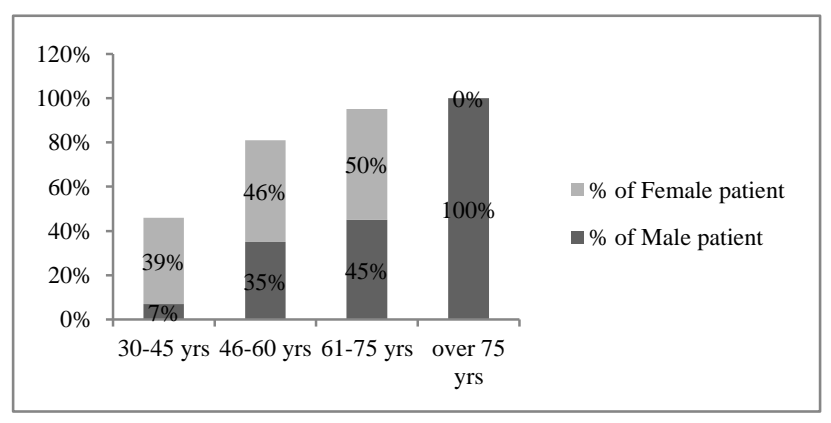

Figure 6: Percentage $(\%)$ of patient of diabetes associated with dyslipidemia. 


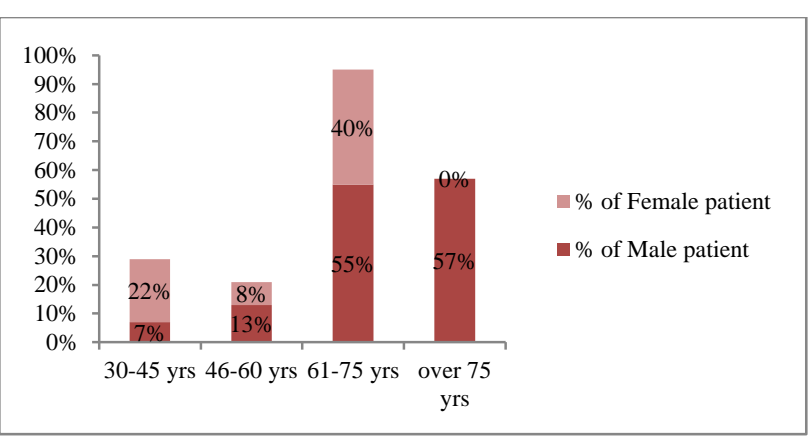

Figure 7: Percentage $(\%)$ of patient of diabetes associated with other complications.

Table 1: Type of anti-diabetic drug contained in prescriptions.

\begin{tabular}{|lll|}
\hline Anti-diabetic drug & Frequency & Frequency (\%) \\
\hline Insulin & 610 & $30.2 \%$ \\
\hline Metformin & 620 & $30.69 \%$ \\
\hline Sitagliptin & 50 & $2.48 \%$ \\
\hline Vildagliptin & 100 & $5 \%$ \\
\hline Linagliptin & 100 & $5 \%$ \\
\hline Glipizide & 40 & $2 \%$ \\
\hline Glimepiride & 110 & $5.45 \%$ \\
\hline Gliclazide & 220 & $10.9 \%$ \\
\hline Pioglitazone & 20 & $1 \%$ \\
\hline $\begin{array}{l}\text { Vildagliptin }+ \\
\text { Metformin }\end{array}$ & 110 & $5.45 \%$ \\
\hline $\begin{array}{l}\text { Sitagliptin }+ \\
\text { Metformin }\end{array}$ & 30 & $1.49 \%$ \\
\hline $\begin{array}{l}\text { Pioglitazone }+ \\
\text { Metformin }\end{array}$ & 10 & $0.5 \%$ \\
\hline
\end{tabular}

In the present study we also found that majority of the patients have diabetes associated with hypertension, dyslipidemia and other complications. To analyze the association of cardiac complications in diabetic patients the present survey was conducted by using a standard questionnaire. A significant portion of the questionnaire highlighted that whether the patients have been diagnosed with any cardiovascular complications and if they have any cardiovascular complications then for how long they have been diagnosed. According to the information of questionnaire it was found that between 30 and 45 years of age $21 \%$ male and $17 \%$ female were diabetic in association with hypertension while $39 \%$ male and $49 \%$ female between the age group of 46 and 60 years suffered from diabetes with hypertension. Moreover, it was observed that $64 \%$ male and $30 \%$ female between 61 and 75 years of age and $86 \%$ male and $0 \%$ female over 75 years of age were diabetic patients with hypertension (Figure 5).

An estimation from the current survey involved $7 \%$ male and $39 \%$ female between 30 and 45 years of age while $35 \%$ male and $46 \%$ female between 46 and 60 years of age have been suffering from diabetes along with dyslipidemia. On the other hand, coexistence of diabetes and dyslipidemia was seen in $45 \%$ male and $50 \%$ female between 61 and 75 years of age while over the age of 75 years all male $(100 \%)$ and no female $(0 \%)$ patients were found to be diabetic in association with dyslipidemia (Figure 6). Furthermore, some of the diabetic patients were significantly found to be in association with other complications for example weakened immune system, oral infections, lung disease, diabetic neuropathy etc. This involved $7 \%$ male and $22 \%$ female between 30 and 45 years of age, $13 \%$ male and $8 \%$ female between 46 and 60 years of age, $55 \%$ male and $40 \%$ female between 61 and 75 years of age and $57 \%$ male over 75 years of age. However, no female $(0 \%)$ diabetic patients were found to be associated with other complications (Figure 7).

\section{DISCUSSION}

A prescription is an order that is written by the physician to tell the pharmacist what medication he/she wants for his/her patients at a particular time in the management of such patients' disorder. Prescriptions given by physician must be containing some elemental components such as name, age of the patient and signature of the prescriber. ${ }^{9}$ To assess and evaluate the prescribing attitude of the physicians and dispensing practice of the pharmacists' prescription based survey is considered the most effective method. ${ }^{10}$

In the present study a total of 1200 prescriptions were assessed and the incidence of Diabetes was seen in 550 (46\%) male and $650(54 \%)$ female patients. The ages of the patients were between 30 and 75 years. Most diabetic patients belong to the age 46-60 years (31\% female, $19 \%$ male) followed by $30-45$ years ( $15 \%$ female, $12 \%$ male) which indicates that in this age group females are mostly affected by diabetes. Almost simillar results were found in a previous study where indicated most patients belonged to the age group of 51-70 years (54.16\%) followed by $30-50$ years $(32.66 \%) .{ }^{11}$ In the patient information section we found that about 2020 drugs were prescribed which belong to 12 different classes. In this case study we found that $30.83 \%$ prescriptions contained single anti-diabetic drugs, $54.16 \%$ contained two antidiabetic drugs and only $15 \%$ contained more than two anti-diabetic drugs. This percentage shows that two antidiabetic drugs were mostly prescribed for diabetic patient. This is known as polypharmacy and may lead to improper use of the prescribed medicines. Similar results were obtained in a previous study. ${ }^{12,13}$ According to Good, (Good 2002) use of multiple medications increases in a variety of ways likelihood of an unintended therapeutic outcome. ${ }^{14}$

In this study we found that most anti-diabetic drugs were prescribed by brand name $(91.66 \%)$ and this percentage is quite high which reflects the strong marketing policy of the pharmaceuticals. Here showed much lower percentage of prescriptions with generic name which is 
similar as previous study that was conducted by WHO within the Indian population. ${ }^{11}$ Among 2020 prescribed anti-diabetic drugs oral anti-diabetic drugs were prescribed in majority of patients (69.80\%), which corresponds with the previous study where $95 \%$ oral antidiabetics were prescribed. ${ }^{15}$

In these prescriptions, about $92.57 \%$ drugs were prescribed as single dose while $7.43 \%$ drugs were prescribed as fixed dose combinations which are similar as the previous study. ${ }^{16}$ Amongst antidiabetic medications, metformin was the most commonly prescribed drug which was given in $30.69 \%$ patients followed by glimepride $10.9 \%, 5.45 \%$ had glipizide, $1 \%$ pioglitazone and other drugs. Similar result was observed in the previous study on prescription pattern. ${ }^{11,15,17}$ For type 2 diabetes mellitus, metformin is reported as the first choice of drug for most patients because metformin has no dangerous effect on cardiovascular risk factors and does not promote weight gain. ${ }^{18}$ On the other hand, a total of $7.43 \%$ drug was prescribed by fixed dose combinations where $5.45 \%$ was Vildagliptin + Metformin combination, $1.49 \%$ was Sitagliptin + Metformin combinatiom and $0.5 \%$ was Pioglitazone + Metformin combination.

From this study we also found that most of the patients have diabetes associated with cardiovascular complications. According to the American Heart Association (AHA) considers diabetes to be one of the seven major controllable risk factors for cardiovascular disease. American Diabetes Association (ADA) observe that there's a big link between diabetes, heart disease, and stroke and claim that at least 68 percent of people age 65 or older with diabetes die from some form of heart disease; and $16 \%$ die of stroke. ${ }^{19}$ Diabetes is treatable, but even when glucose levels are under control it greatly increases the risk of heart disease and stroke. ADA also considers that patients with type 2 diabetes may have hypertension, dyslipidemia, obesity that contributes to their risk for developing cardiovascular disease. To find out the correlation of diabetic patients with cardiovascular complications a questionnaire form was used.

Diabetes mellitus and hypertension are interrelated diseases that strongly predispose an individual to atherosclerotic cardiovascular disease. ${ }^{20,21}$ Generally most of the patients are diabetic in association with hypertension. According to the information found in the questionnaire, most of the male patients (64\%) were diabetic in association with hypertension between the age group of 61 and 75 years. On the other hand, between the age group of 46 and 60 years most of the female patients (49\%) were diabetic in association with. Moreover, it was observed that $0 \%$ female over 75 years of age were diabetic in association with hypertension. This result shows that after 75 years of age female patients are not vulnerable for diabetes in association with hypertension.
Another cardiovascular complication known as dyslipidemia is also a common disease for diabetic patients. Dyslipidemia, an established risk factor for CVD, is strikingly common in patients with type 2 diabetes, affecting almost $50 \%$ of this population. ${ }^{22}$ After analyzing the questionnaire we found that almost all patients had diabetes in association with dyslipidemia. It was observed that at the age between 30 and 45 years $7 \%$ male and $39 \%$ female, at the age between 46 and 60 years $35 \%$ male and $46 \%$ female and the age between 61 and 75 years $45 \%$ male and $50 \%$ female patients were found to be diabetic in association with dyslipidemia while over the age of 75 years all male $(100 \%)$ and no female $(0 \%)$ patients were found to be diabetic in association with dyslipidemia.

Furthermore, in this study we also found that some diabetic patients had complications other than hypertension and dyslipidemia such as weakened immune system, oral infections, lung disease, diabetic neuropathy etc. In this case after 75 years most of the male patients (57\%) were found to be associated with other complications. On the other hand most of the female patients (40\%) were found to be associated with other complications.

\section{CONCLUSION}

Oral anti-diabetic drugs were advised in majority, which is easier method of drug administration. From the present study based on collected data from prescriptions, we found that majority of patients were affected by type- 2 diabetes with cardiovascular complications. To treat these diabetic patients anti-diabetic drugs have been prescribed irrationally where metformin $(30.69 \%)$ was found to be most commonly prescribed anti-diabetic drug. Furthermore, our study reported that the female patients $(54 \%)$ were significantly more affected by diabetes associated with cardiovascular complications than male patients. To reduce morbidity and mortality in diabetic patients, we must ensure optimum glycemic control not only by prescribing keeping in line with guidelines, but also by ensuring patients' adherence to treatment plan.

\section{Funding: No funding sources \\ Conflict of interest: None declared}

Ethical approval: The study was approved by the Institutional Ethics Committee

\section{REFERENCES}

1. Michael H. Statin/fibrate combination in patients with metabolic syndrome or diabetes: evaluating the risks of pharmacokinetic drug interactions. Expert Opin. Drug. 2006;5(1):145-56.

2. DeFronzo RA, bonadonna RC, Ferrannini E. Pathogenesis of NIDDM: A balanced overview. Diabetes Care. 2002;15:318-68.

3. Alessandra, Lucianne, Roberta, Catia C, Carlos, Brito G. Impact of Diabetes on Cardiovascular Disease: Int. J. Hyper. 2013:2-5. 
4. Diabetes in America, National Institutes of Health, National Institute of diabetes and Digestive and Kidney Diseases, Bethesda, MD, USA, 2nd edition, 1995.

5. Amanda NL, Samuel DJ. The Comorbidities of Diabetes and Hypertension: Mechanisms and Approach to Target Organ Protection. J Clin Hypertens. 2011;13(4):244-6.

6. Arshag DM. Management of type 2 diabetes mellitus Apharmacoepidemiology. 2009:150-159.

7. Rafael C. Type 2 diabetes, dyslipidemia, and vascular risk: Rationale and evidence for correcting the lipid imbalance. American heart journal. 2005;150:859-70.

8. Alvin C. Harrison's Principles of Internal Medicine; Diabetes Mellitus, 1995:345.

9. Practitioner's manual: Valid prescription requirements. U. S. Department of justice, Drug enforcement administration. 2006 Edition.

10. Yuen YH, Chang S, Chong CK, Lee SC, Critchlev JA, Chan JC. Drug utilization in a hospital general medical outpatient clinic with particular reference to antihypertensive and antidiabetic drugs. J Clin Pharm Ther. 1998;23:287-94.

11. Ramachandran G, Rohith V, Isabella Topno. Evaluation of prescribing pattern of anti-diabetic drugs using WHO prescribing indicators in a tertiary care hospital in Puducherry: A cross-sectional study. The Pharma Innovation Journal 2015;4(5):76-80.

12. Alebiosu CO. Antidiabetics/Antihypertensives prescription profiles in OSUTH, Sagamu and environment. Nigerian J Clin Prac. 2004;7(1):15-20.

13. Eze, Ojieabu. Pattern of Prescriptions among Elderly Diabetic- Hypertensive Patients in a Nigerian Teaching Hospital. Afr. J. Biomed. Res. 2010;13(3):169-73.

14. Good CB. Polypharmacy in Elderly patients with diabetes. Diabetes Spectrum. 2002; 15:240-248.
15. Vengurlekar S, Shukla P, Patidar P, Bafna R, Jain S. Prescribing pattern of antidiabetic drugs in Indore city hospital. Indian J Pharm Sci. 2008;70:637-40.

16. Shaktibala D, Mirza Atif B, Mohammad A, Amit V, Shalu B. Study of prescribing pattern in diabetes mellitus patients in a tertiary care teaching hospital at dehradun, uttarakhand. Int $\mathbf{J}$ Med Sci Pub $\mathrm{H}$. 2014;3(11):1-8.

17. Dhanraja E, Raval AD, Yadav R, Bhansali A, Tiwari P. Prescribing pattern of antidiabetic drugs and achievement of glycemic control in T2DM patients in tertiary care hospital in North India. Int J Diab Dev C. 2013:33:140-6.

18. Krentz AJ, Bailey CJ. Oral antidiabetic agents: Current role in type 2 diabetes mellitus. Drugs. 2005;65:385411.

19. Grundy SM, Cleeman JI, Daniels SR, Donato KA, Eckel RH, Franklin BA, Gordon DJ, Krauss RM, Savage PJ, Smith SC, Spertus JA, Costa F, Diagnosis and Management of the Metabolic Syndrome: An American Heart Association/National Heart, Lung, and Blood Institute Scientific Statement' Circulation. 2005;112(17):2735-52.

20. Epstein M, Sowers JR. Diabetes mellitus and hypertension. Hypertension. 1992;19:403-18.

21. The National High Blood Pressure Education Program Working Group. National High Blood Pressure Education Program Working Group report on hypertension in diabetes. Hypertension. 1994;23:14558.

22. Saydah SH, Fradkin J, Cowie CC. Poor control of risk factors for vascular disease among adults with previously diagnosed diabetes. JAMA. 2004;291:33542.

Cite this article as: Karim $\mathrm{R}$, Saha $\mathrm{R}$, Sohanur Rahman M, Nure A, Etu KA, Jamila U, et al. A survey of prescription pattern of anti-diabetic drugs on diabetic patients with cardiovascular complications within Dhaka metropolis. Int J Basic Clin Pharmacol 2016;5:2397-2402. 